\title{
TALEN-based generation of a cynomolgus monkey disease model for human microcephaly
}

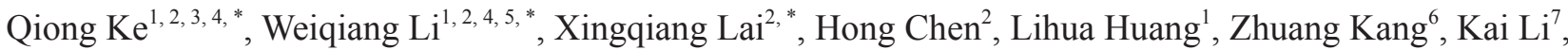
Jie Ren ${ }^{7}$, Xiaofeng Lin ${ }^{8}$, Haiqing Zheng, Weijun Huang ${ }^{2}$, Yunhan $\mathrm{Ma}^{10}$, Dongdong $\mathrm{Xu}^{10}$, Zheng Chen ${ }^{11}$, Xinming Song ${ }^{11}$, Xinyi Lin $^{11}$, Min Zhuang ${ }^{12}$, Tao Wang ${ }^{2,5}$, Fengfeng Zhuang ${ }^{13}$, Jianzhong Xi ${ }^{14}$, Frank Fuxiang Mao ${ }^{15}$, Huimin Xia ${ }^{1}$, Bruce T Lahn ${ }^{16}$, Qi Zhou ${ }^{17}$, Shihua Yang ${ }^{10}$, Andy Peng Xiang ${ }^{1,2,4,5}$

${ }^{I}$ Program of Stem Cells and Regenerative Medicine, Affiliated Guangzhou Women and Children's Hospital, Zhongshan School of Medicine, Sun Yat-Sen University, Guangzhou 510623, China; ${ }^{2}$ Center for Stem Cell Biology and Tissue Engineering, Key Laboratory for Stem Cells and Tissue Engineering, Ministry of Education, Sun Yat-Sen University, Guangzhou 510080, China; ${ }^{3}$ Department of Biology, Zhongshan Medical School, Sun Yat-Sen University, Guangzhou 510080, China; ${ }^{4}$ Guangdong Key Laboratory of Reproductive Medicine, Guangzhou 510080, China; ${ }^{5}$ Department of Biochemistry, Zhongshan Medical School, Sun Yat-Sen University, Guangzhou 510080, China; ${ }^{6}$ Department of Radiology, the Third Affiliated Hospital of Sun Yat-Sen University, Guangzhou 510632, China; ${ }^{7}$ Department of Ultrasound, the Third Affiliated Hospital of Sun Yat-sen University, Guangzhou 510632, China; ${ }^{8}$ Department of Radiology, Sun Yat-Sen Memorial Hospital, Sun Yat-Sen University, Guangzhou 510120, China; ${ }^{9}$ Department of Rehabilitation Medicine Science, the Third Affiliated Hospital of Sun Yat-sen University, Guangzhou 510632, China; ${ }^{10}$ College of Veterinary Medicine, Guangdong Provincial Key Laboratory of Prevention and Control for Severe Clinical Animal Diseases, South China Agricultural University, Guangzhou 510642, China, ${ }^{11}$ Department of Medical Genetics, Zhongshan School of Medicine, Sun Yat-sen University, Guangzhou 510080, China; ${ }^{12}$ School of Life Science and Technology, ShanghaiTech University, Shanghai 200031, China, ${ }^{13}$ Beijing ViewSolid Biotechnology, Beijing 100034, China: ${ }^{14}$ Department of Biomedical Engineering, College of Engineering, Peking University, Yannan Yuan 60, Beijing 100871, China; ${ }^{15}$ State Key Laboratory of Ophthalmology, Zhong Shan Ophthalmic Center, Sun Yat-sen University, Guangzhou 510060, China; ${ }^{16}$ Department of Human Genetics, University of Chicago, Chicago, IL 60637, USA; ${ }^{17}$ State Key Laboratory of Stem cell and Reproductive Biology, Institute of Zoology, Chinese Academy of Sciences, Beijing 100101, China

Gene editing in non-human primates may lead to valuable models for exploring the etiologies and therapeutic strategies of genetically based neurological disorders in humans. However, a monkey model of neurological disorders that closely mimics pathological and behavioral deficits in humans has not yet been successfully generated. Microcephalin 1 (MCPH1) is implicated in the evolution of the human brain, and MCPH1 mutation causes microcephaly accompanied by mental retardation. Here we generated a cynomolgus monkey (Macaca fascicularis) carrying biallelic $\mathrm{MCPH} 1$ mutations using transcription activator-like effector nucleases. The monkey recapitulated most of the important clinical features observed in patients, including marked reductions in head circumference, premature chromosome condensation (PCC), hypoplasia of the corpus callosum and upper limb spasticity. Moreover, overexpression of $M C P H 1$ in mutated dermal fibroblasts rescued the PCC syndrome. This monkey model may help us elucidate the role of $\mathrm{MCPH} 1$ in the pathogenesis of human microcephaly and better understand the function of this protein in the evolution of primate brain size.

Keywords: $M C P H 1$; microcephaly; TALEN; gene mutant; cynomolgus monkey

Cell Research (2016) 26:1048-1061. doi:10.1038/cr.2016.93; published online 9 August 2016

*These three authors contributed equally to this work.

Correspondence: Andy Peng Xiang ${ }^{\mathrm{a}}$, Shihua Yang ${ }^{\mathrm{b}}$, Qi Zhou ${ }^{\mathrm{c}}$

a'E-mail: xiangp@mail.sysu.edu.cn

bE-mail: yangsh@scau.edu.cn

'E-mail: qzhou@ioz.ac.cn

Received 9 March 2016; revised 23 May 2016; accepted 27 May 2016; published online 9 August 2016

\section{Introduction}

Human pluripotent stem cells can be used to develop various in vitro organoid culture systems, including cerebral organoid, which may be used to understand the mechanisms involved in organogenesis and disease $[1,2]$. 
However, the human brain contains over 170 billion neurons and non-neuronal cells distributed throughout various regions [3]. In vitro organogenesis remains insufficient to model human neurological and mental disorders. In recent decades, gene-edited animal models, especially rodent models, have been generated and used to improve our understanding of pathogenic mechanisms and to test potential treatments for human neurological diseases. However, given the myriad behavioral, physiological and anatomical differences between humans and rodents, many rodent models fail to faithfully recapitulate the human condition [4-6]. Because non-human primates have perceptual, cognitive and behavioral repertoires very similar to those of humans, the development of non-human primate models that mimic human neurological diseases would critically contribute to our understanding of the mechanisms at work in the human brain $[7,8]$.

Autosomal recessive primary microcephaly $(\mathrm{MCPH})$ is a neurodevelopmental disorder that is defined by the presence of a head circumference that is three standard deviations below the mean ( $-3 \mathrm{SD})$ for age and sex [9-11]. More than 12 causative genes have been identified; the first of which was designated as $M C P H 1$ (microcephalin 1; also known as BRIT1 for BRCT-repeat inhibitor of human telomerase reverse transcriptase (hTERT) expression) $[12,13]$. Evolutionary studies have suggested that changes in the $\mathrm{MCPH} 1$ gene played an important role in the enlargement of the human brain. Moreover, this gene demonstrates evidence of positive selection during the evolution of humans and great apes [14-16]. Truncations in the $M C P H 1$ gene are associated with significantly reduced brain size and short stature $[12,17,18]$. Some patients exhibit mental retardation and other neurological conditions, including spasticity [18-20]. Magnetic resonance imaging (MRI) revealed the presence of cerebral malformations, such as a simplified gyral pattern in the brain and hypoplasia of the corpus callosum [18, 21-23]. Several elegant studies have demonstrated that MCPH1-deficient mice could recapitulate some key features of microcephaly and could thus help us understand the pathogenic mechanism. For example, Chen et al. reported $\mathrm{MCPH1}$-deficient mice exhibited small skull sizes and hearing impairment [24]. Another group also found disruption of $\mathrm{MCPHI}$ caused microcephaly due to a premature switching of neuroprogenitors from symmetric to asymmetric division [25]. So far, no neurologic symptoms have been found in mouse models and not all MCPH1 mutant mice recapitulate the severely reduced brain size observed in human patients $[26,27]$. Thus, a nonhuman primate model is desirable.

Genetically modified monkey models for human diseases, such as a transgenic Huntington's disease monkey model and an autism-related gene $M E C P 2$ transgenic monkey model, have been created and were thought to mimic human behavior and cognitive phenotypes better than rodents $[28,29]$. However, precise gene editing in monkeys is still a time-consuming and difficult undertaking. More recently, several studies have improved upon the techniques using transcription activator-like effector nucleases (TALENs) or clustered regularly interspaced short palindromic repeats/Cas9 [30-33]. Two groups reported the generation of $M E C P 2$ knockout monkey models using TALENs $[30,31]$. However, it is not clear whether the TALEN-mediated gene mutagenesis can effectively disrupt the targeted gene function and recapitulate disease phenotypes in non-human primates. Here we report a TALEN-generated $M C P H 1$ mutant cynomolgus monkey and the phenotypic characterization of this potential monkey model of MCPH.

\section{Results}

\section{Generation of the MCPH1 mutant monkey}

Similar to the human gene, the monkey $\mathrm{MCPH} 1$ gene comprises 14 exons and encodes an 842 amino acid protein, which contains three BRCT (BRCA1 C terminus) domains playing the diverse roles of MCPH1 by mediating its interactions with distinct partners [9]. We designed three TALEN pairs targeting different sites inside exons 2 and 3 (MCPH1-T1 T3), which encode the $\mathrm{N}$-terminal BRCT1 domain (Figure 1A).

The targeting efficiency of TALEN pairs was examined in COS-7 cells using the T7 endonuclease 1 (T7EN1) assay; all three TALEN pairs could introduce mutations in the $M C P H 1$ locus. The best indel rate approached $45.2 \%$. Sanger sequencing revealed that MCPH1-T2 yielded the highest mutation rate ( 8 of 24 sequencing reads were mutated; 33\%) in its target site, indicating that this TALEN pair could efficiently introduce genomic mutations in the $\mathrm{MCPH1}$ gene. The targeting efficiencies of all three TALEN pairs are presented in Supplementary information, Figure S1A.

To further test the targeting efficiency of $M C P H 1-\mathrm{T} 2$ in monkey embryos, we injected the $M C P H 1$-T2 mRNA at two concentrations $(20 \mathrm{ng} / \mu \mathrm{l}$ and $50 \mathrm{ng} / \mu \mathrm{l})$ into the cytoplasm of 23 cynomolgus monkey zygotes obtained by intracytoplasmic sperm injection (ICSI). Although Sanger sequencing revealed that $85.7 \%$ of embryos injected with $50 \mathrm{ng} / \mu 1 \mathrm{mRNA}$ were mutant, only $50 \%$ of the embryos developed to the morula/blastocyst stage (Supplementary information, Figure S1B-S1D). In the lower concentration group, the mutation rate was $50 \%$, and the embryos were of better quality, with $62.5 \%$ developing to the morula/blastocyst stage. As these results 
indicate that $20 \mathrm{ng} / \mu \mathrm{l} M C P H 1-\mathrm{T} 2$ could produce mutations in monkey embryos with acceptable efficiency and low toxicity, this was used for the subsequent experiments.

We injected 55 embryos with $M C P H 1$-T2 mRNA and transferred 52 embryos into nine surrogate female monkeys (Figure 1B). Three surrogates became pregnant. One experienced a miscarriage at day 46 after embryo transfer. The aborted embryo was not collected. The re- maining two females delivered three offspring upon the completion of pregnancy ( $\sim 165$ days).

\section{Genotype analysis of the MCPHI mutant monkey}

T7EN1 assays and Sanger sequencing both revealed that the twin offspring were wild type (Supplementary information, Figure S2A and S2B). In contrast, the singleton offspring (female) carried biallelic $\mathrm{MCPH} 1$ gene mutations at the targeting site and was designated

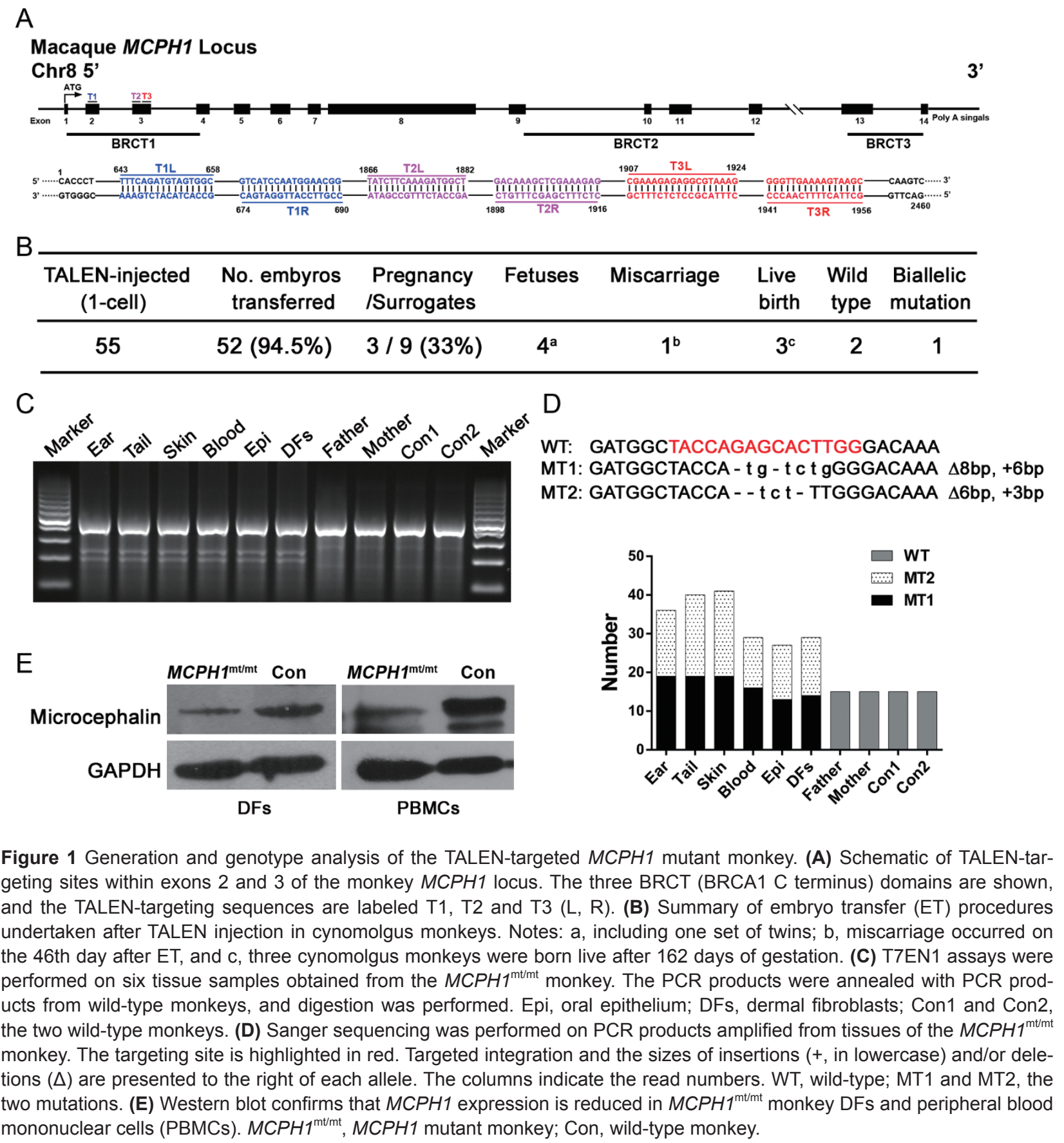


as the $M C P H 1^{\mathrm{mt} / \mathrm{mt}}$ monkey. Samples from the ear, tail, skin, blood, oral epithelium (Epi) and dermal fibroblast cells (DFs) of the $\mathrm{MCPHI}^{\mathrm{m} t \mathrm{mt}}$ monkey could be cleaved by T7EN1, whereas those of its parents and the controls could not be cleaved (Figure 1C). These results demonstrate that the $M C P H 1^{\mathrm{mt} / \mathrm{mt}}$ monkey contains mutations in the $M C P H 1$ sequence. Further sequencing of the TALEN-targeting site of the $M C P H 1^{\mathrm{mt} / \mathrm{mt}}$ monkey revealed the presence of two different mutations in the MCPH1 gene (Figure 1D): an 8-bp deletion together with a 6-bp insertion that resulted in a nonsense mutation and a 6-bp deletion with a 3-bp insertion that resulted in a deletion/missense mutation. These two alterations were observed in all tested tissue samples at a $\sim 1: 1$ ratio. We selected $>200$ colonies and failed to identify any wildtype colony upon sequencing. We performed PCR with a primer pair that could amplify the wild-type allele but not the mutant allele to detect more sensitively the biallelic mutations of the $M C P H 1$ gene, and did not find the wild-type $M C P H 1$ allele in the $M C P H 1^{\mathrm{mt} / \mathrm{mt}}$ monkey (Supplementary information, Figure S2C). These results demonstrate that the $M C P H 1^{\mathrm{mt} / \mathrm{mt}}$ monkey carries biallelic mutations in the $M C P H 1$ gene. Parents of the $M C P H 1^{\mathrm{mt} / \mathrm{mt}}$ monkey contain only the wild-type $M C P H 1$ allele. This result confirms that the mutations in the offspring were generated by TALEN-mediated mutagenesis.

Because TALENs may also cause off-target mutations $[34,35]$, we searched for any potential off-target mutations in the $M C P H 1^{\mathrm{mt} / \mathrm{mt}}$ monkey. TALENoffer computational prediction tool [36] was used to predict the possible off-target sites (OTSs) in the Macaca fascicularis genome. Thirteen potential OTSs with TALENoffer score $>-1.61$ were chosen, and no mutation was detected by T7EN1 assays and Sanger sequencing in these sites in the $M C P H I^{\mathrm{mt} / \mathrm{mt}}$ monkey. Because these potential OTSs were all intronic loci, we next tested additional 18 potential OTSs located in exons with TALENoffer score $>$ -1.98 and found no mutation in these sites (Supplementary information, Figure S2D and Table S1). The frameshift and deletion/missense mutations in the $M C P H 1^{\mathrm{mt} / \mathrm{mt}}$ monkey genome were predicted to generate a truncated protein $(75 \mathrm{aa})$ and a protein missing one amino acid (p.57QST $>$ HL), respectively (Supplementary information, Figure S2E). Western blot revealed that microcephalin was expressed at lower levels in the $M C P H I^{\mathrm{mt} /}$ ${ }^{\mathrm{mt}}$ monkey DFs and peripheral blood mononuclear cells (PBMCs) compared with controls (Figure 1E).

MCPH1 mutant monkey exhibited a visibly smaller brain

Humans with $M C P H 1$ mutations are characterised by significant reductions in head circumference and may exhibit shortened height $[12,17,18]$. To assess the phe- notype of the $M C P H 1^{\mathrm{mt} / \mathrm{mt}}$ monkey, four age-matched wild-type female cynomolgus monkeys were selected as controls. The female $M C P H 1^{\mathrm{m} t / \mathrm{mt}}$ monkey exhibited a visibly smaller brain (head) and a slightly shorter stature than the wild-type monkeys (Figure 2A). Noticeable microcephaly was observed at birth, with an occipital-frontal circumference (OFC) of $15.3 \mathrm{~cm}(-5 \mathrm{SD}$, mean \pm SD of controls: $17.3 \pm 0.4 \mathrm{~cm})$. The monkey's body length at birth was average at $16.2 \mathrm{~cm}(-2.3 \mathrm{SD}$, mean \pm SD of controls: $17.4 \pm 0.5 \mathrm{~cm})$, whereas the body weight at birth was only $0.241 \mathrm{~kg}(-5 \mathrm{SD})$, which was much lower than that of wild-type monkeys (mean \pm SD: $0.390 \pm 0.030 \mathrm{~kg}$ ) of the same age and sex. Longitudinal measurement of OFC, body weight, body length, tail length and chest circumference revealed a slower growth trajectory of the $M C P H 1^{\mathrm{mt} / \mathrm{mt}}$ monkey (Figure 2B). The OFC of the $M C P H 1^{\mathrm{m} t \mathrm{mt}}$ monkey was below normal (4 to -11 SD) during the first year of life. The weight of the $M C P H 1^{\mathrm{m} t / \mathrm{mt}}$ monkey was only $\sim 65 \%$ compared with wild-type animals before 6 months of age and below normal ( -2 to $-8 \mathrm{SD})$ during the first year of life. The differences between the $M C P H 1^{\mathrm{mt} / \mathrm{mt}}$ monkey and controls in body length, tail length and chest measurement were also significant. In contrast, monthly examinations of the teeth revealed no significant difference between the $M C P H I^{\mathrm{m} t / \mathrm{mt}}$ monkey and controls, with all teeth erupting before 6 months of age.

Because previous studies demonstrated that $\mathrm{MCPH1}$ mutant patients may exhibit a simplified gyral pattern, hypoplasia of the corpus callosum and frontal lobe [18, $21,37]$, we performed MRI to detect the brain size of the $M C P H 1^{\mathrm{mt} / \mathrm{mt}}$ monkey. The total brain volume (TBVs) was $41.9 \mathrm{ml}$ in the $M C P H I^{\mathrm{m} t / \mathrm{mt}}$ monkey compared with 67.6 $\pm 3.3 \mathrm{ml}$ (mean $\pm \mathrm{SD}$ ) in wild-type monkeys at 6 -month old ( $-8 \mathrm{SD}$; Figure $2 \mathrm{~B})$. The corpus callosum formed but appeared thin for the animal's age, most notably in the region of the body (Figure $2 \mathrm{C}(\mathrm{a}-\mathrm{c}, \mathrm{e}-\mathrm{g})$ ). In addition to hypoplasia of the corpus callosum, MRI did not detect any other obvious malformations in the brain of the $M C P H 1^{\mathrm{mt} / \mathrm{mt}}$ monkey (Figure $2 \mathrm{C}(\mathrm{d}, \mathrm{h})$ ). These results suggest that the phenotype of the $M C P H 1^{\mathrm{mt} / \mathrm{mt}}$ monkey is similar to the symptoms of $M C P H 1$ patients.

\section{Behavior and neurologic changes in the MCPH1 mutant monkey}

Neitzel et al. reported a $M C P H 1$ mutant patient who was unable to walk without support until 4.75 years [18], whereas another report described a patient with delayed acquisition of fine motor skills [19]. The $M C P H 1^{\mathrm{m} t \mathrm{mt}}$ monkey walked freely without any assistance similar to the controls; however, the $M C P H 1^{\mathrm{mt} / \mathrm{mt}}$ monkey had occasional spasms (Supplementary information, Movie 

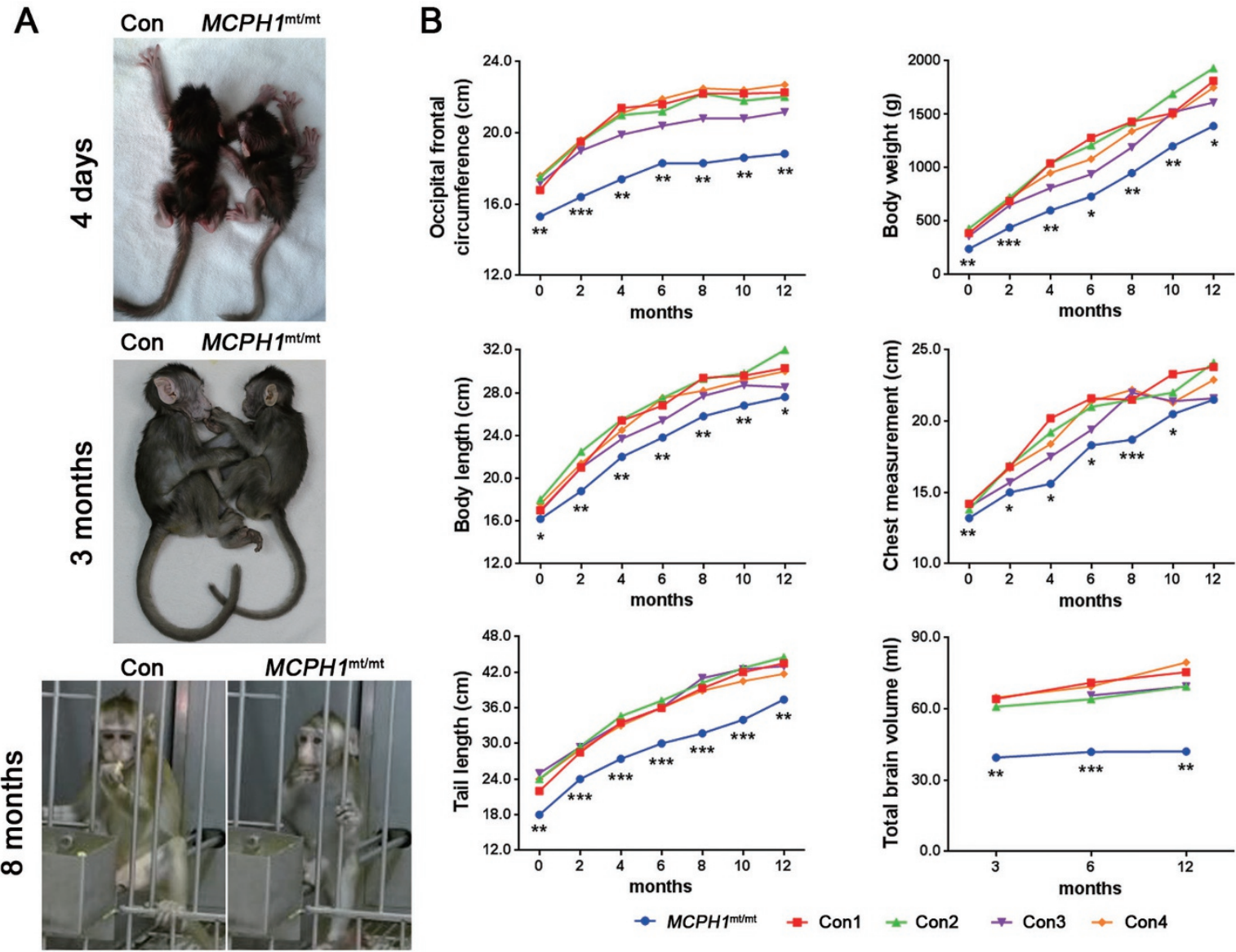

C

3 months

6 months

12 months

12 months
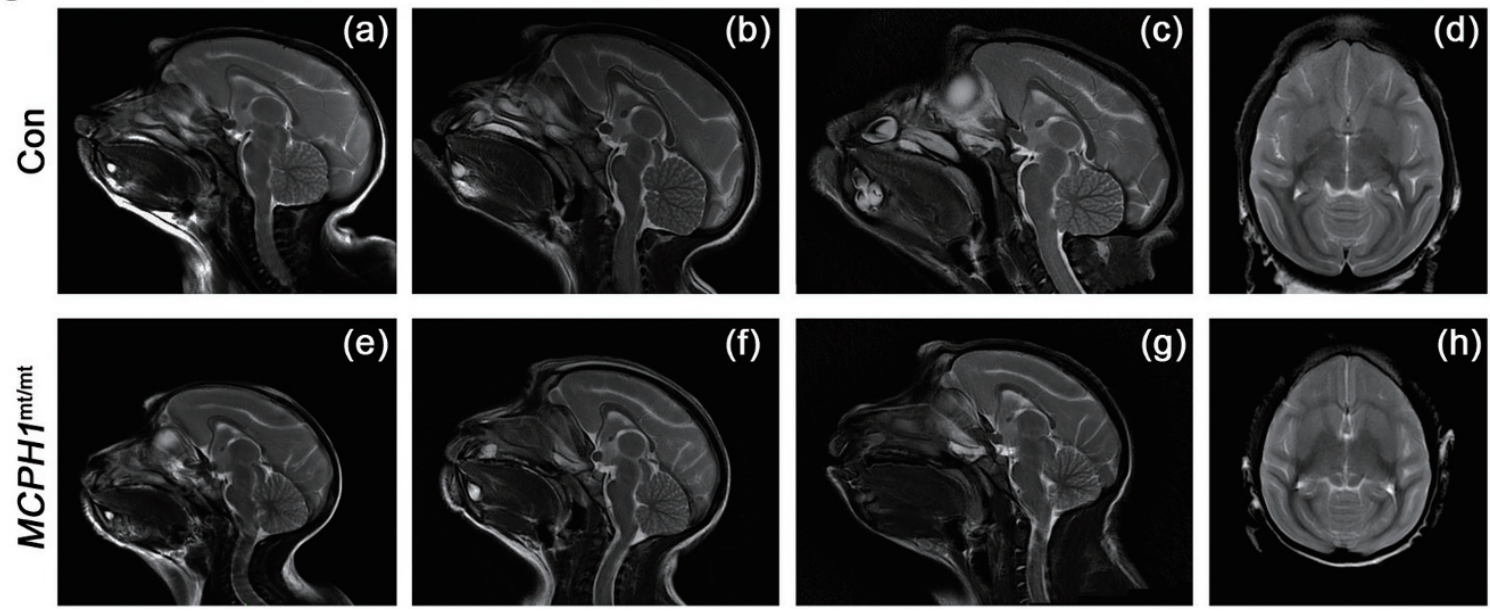

Figure 2 Physical measurements and MRI-based phenotypes of the TALEN-targeted MCPH1 mutant monkey. (A) Images of the $M C P H 1^{\mathrm{mt} / \mathrm{mt}}$ monkey and a wild-type monkey of the same age and sex. The MCPH1 mutant monkey exhibits a visibly smaller head and shorter stature. (B) Longitudinal measurements of the occipital frontal circumference, body weight, body length, tail length and chest circumference. Total brain volumes were calculated using MRI. Con 1-4, four wild-type monkeys of the same age and sex. All data are represented as the mean $\pm \mathrm{SD}, n=3$ and ${ }^{\star} P<0.05,{ }^{* \star} P<0.01$, ${ }^{* \star *} P<0.001$ versus wild-type monkeys (Student's $t$-test). (C) MRI images show the typical reduction of brain volume of the $M C P H 1^{\mathrm{mt} / \mathrm{mt}}$ monkey $(\mathrm{e}-\mathrm{h})$ in comparison to an age- and sex-matched control (a-d). Sagittal T2-weighted images indicating that the corpus callosum of the $\mathrm{MCPH} 1^{\mathrm{mt} / \mathrm{mt}}$ monkey $(\mathrm{e}-\mathrm{g})$ is thinner than that of a wild-type monkey (a-c). Axial T2-weighted images displaying most of the brain anatomy is normal except for the size in the $M C P H 1^{\mathrm{mt} / \mathrm{mt}}$ monkey (h) compared with wild-type monkeys (d). Con, wildtype monkey; $M C P H 1^{\mathrm{mt} / \mathrm{mt}}, M C P H 1$ mutant monkey. 
S1) and often could not jump or climb as flexibly as the controls (Supplementary information, Movie S2). We evaluated the videos at random and calculated the success rates of jumps made by wild-type and $M C P H 1^{\mathrm{mt} /}$ ${ }^{\mathrm{mt}}$ monkeys over $30 \mathrm{~min}$. The total number of times the $M C P H 1^{\mathrm{mt} / \mathrm{mt}}$ monkey attempted to jump did not differ from the wild-type monkeys. However, the former succeeded only one-seventh of the time, whereas the controls never failed (Figure 3A). Notably, the $M C P H I^{\mathrm{mt} / \mathrm{mt}}$ monkey rarely hung upside down, whereas the wild-type monkeys did so once approximately every $4 \mathrm{~min}$. The wild-type monkeys hung upside down 3-13 times within $30 \mathrm{~min}$, whereas the $M C P H 1^{\mathrm{mt} / \mathrm{mt}}$ monkey did so only once within $180 \mathrm{~min}$ (from a total of six random videos; Figure 3B).

In addition to the above results, the $M C P H 1^{\mathrm{mt} / \mathrm{mt}}$ monkey's arms could not be fully extended under external force, even when the animal was anesthetized (Figure $3 \mathrm{C}$ ), which was similar to the previous observation that a $M C P H 1$ patient demonstrated spasticity with hyperreflexia of the patellar and Achilles tendon reflexes and clonus [18]. Radiological examination of the $M C P H 1^{\mathrm{mt} /}$ ${ }^{\mathrm{mt}}$ monkey did not find any bone deformity (Figure 3D). Thus, we used shear-wave elastography to quantify biceps brachii stiffness at a $90^{\circ}$ fixed position [38]. Among the control monkeys, the mean elasticity values of the right biceps brachii muscle ranged from 9.90 to 12.20 $\mathrm{kPa}$, whereas those of the left ranged from 9.95 to 12.38 $\mathrm{kPa}$. In contrast, in the $M C P H 1^{\mathrm{mt} / \mathrm{mt}}$ monkey, these values averaged $19.12 \pm 1.37$ and $20.77 \pm 0.75 \mathrm{kPa}$ (mean \pm SEM), respectively. The biceps brachii of the $M C P H 1^{\mathrm{mt} /}$ ${ }^{\mathrm{mt}}$ monkey had significantly greater stiffness than the controls, as confirmed by one-way ANOVA (Figure 3E). This result suggests that the introduced $M C P H 1$ mutation may affect the motor skills of the mutant monkey in a manner similar to that observed in some patients.

\section{Cellular phenotype of the MCPH1 mutant monkey}

$M C P H 1$-related microcephaly has also been associated with premature chromosome condensation (PCC) syndrome [17, 18, 39, 40], in which PCC occurs at interphase, resulting in a high number of cells with prophase-like chromatin. To investigate whether cells of the $M C P H I^{\mathrm{mt} / \mathrm{mt}}$ monkey exhibited evidence of PCC, we examined chromosome preparation made from DFs. Markedly more nuclei containing condensed prophaselike chromatin were found in the DFs of the $M C P H I^{\mathrm{mt}}$ ${ }^{\mathrm{mt}}$ monkey (mean \pm SEM: $11.5 \% \pm 0.7 \%$ ) than in the wild-type cells (mean \pm SEM: $0.9 \% \pm 0.1 \%$; Figure $4 \mathrm{~A}$ ). To investigate whether the nuclear envelope was retained or disassembled in PCC in the prophase-like cells (PLCs), the expression of Lamin B in DFs was detected by immunofluorescence staining, and the chromatin was stained with DAPI (Figure 4B). PLCs with intact nuclear envelopes were relatively common among the DFs of the $M C P H I^{\mathrm{mt} / \mathrm{mt}}$ monkey (mean \pm SEM: $12.0 \%$ $\pm 1.1 \%$ ), but not in the controls (mean \pm SEM: $1.30 \%$ $\pm 0.2 \%)$. Collectively, these data demonstrate that the $M C P H 1^{\mathrm{m} t \mathrm{mt}}$ monkey presents typical cellular symptoms of $M C P H 1$-mutant microcephalus patients.

As $M C P H 1$ is a BRCT-repeat protein capable of inhibiting hTERT expression [41, 42], we examined whether TERT was inhibited in $M C P H 1^{\mathrm{mt} / \mathrm{mt}}$ cells. We used the telomeric repeat amplification protocol (TRAP) to assess telomerase enzymatic activity and found that telomerase activity was significantly activated in $M C P H 1^{\mathrm{mt} / \mathrm{mt}} \mathrm{DFs}$ compared with wild-type DFs $(P<0.001$; Figure 4C).

A previous report revealed that normal prophase cells decondense their chromatin to allow repair following DNA damage, but MCPH1 patient cells do not [43, 44]. We examined whether ionizing irradiation (IR) affected the chromosome condensation behavior of $M C P H 1^{\mathrm{mt} / \mathrm{mt}}$ DFs compared with controls. Cultured DFs were subject to $1 \mathrm{~Gy}$ irradiation, and cytological preparations were made at 2-h intervals for $8 \mathrm{~h}$ following IR before chromatin morphology was analyzed. We found that $M C P H 1^{\mathrm{mt} /}$ ${ }^{\mathrm{mt}}$ DFs failed to completely decondense their chromatin following DNA damage. The number of PLCs remained relatively constant at all time points in $M C P H I^{\mathrm{mt} / \mathrm{mt}} \mathrm{DFs}$, whereas relatively fewer PLCs were found in control DFs (Figure 4D). Therefore, the chromosome condensation behavior of $M C P H 1$-mutant DFs remained after DNA damage.

To confirm that the phenomena observed in vitro were due to $\mathrm{MCPH} 1$ mutations, an $\mathrm{MCPH1}$-expressing lentiviral vector was established and introduced into $M C P H 1^{\mathrm{mt} / \mathrm{mt}}$ monkey DFs to generate $M C P H 1^{\text {rescue }} \mathrm{DFs}$ (Supplementary information, Figure S3A). As a control, $M C P H 1^{\mathrm{mt} / \mathrm{mt}}$ monkey DFs were transduced with a mock vector (encoding eGFP alone) to generate $M C P H 1^{\text {mock }}$ DFs. After viral infection, $>90 \%$ of the DFs were infected, as reflected by GFP fluorescence (Figure 4E). Western blot revealed that microcephalin expression was significantly upregulated in $M C P H I^{\text {rescue }}$ DFs compared with the low level observed in $M C P H 1^{\text {mock }}$ DFs (Supplementary information, Figure S3B). An analysis of chromosome morphology revealed that the proportion of PLCs in $M C P H 1^{\text {rescue }}$ DFs (mean \pm SEM: $1.8 \% \pm 0.2 \%$ ) was reduced markedly compared with $M C P H 1^{\text {mock }} \mathrm{DFs}$ (mean \pm SEM: $11.1 \% \pm 1.0 \%$ ) to a level similar to that observed in wild-type cells (Figure 4E and 4F). The TRAP test also revealed that $M C P H{ }^{\text {rescue }}$ DFs could inhibit TERT expression to the same degree as in controls (Figure 4G). These data indicate that ectopic expression of $\mathrm{MCPH1}$ 

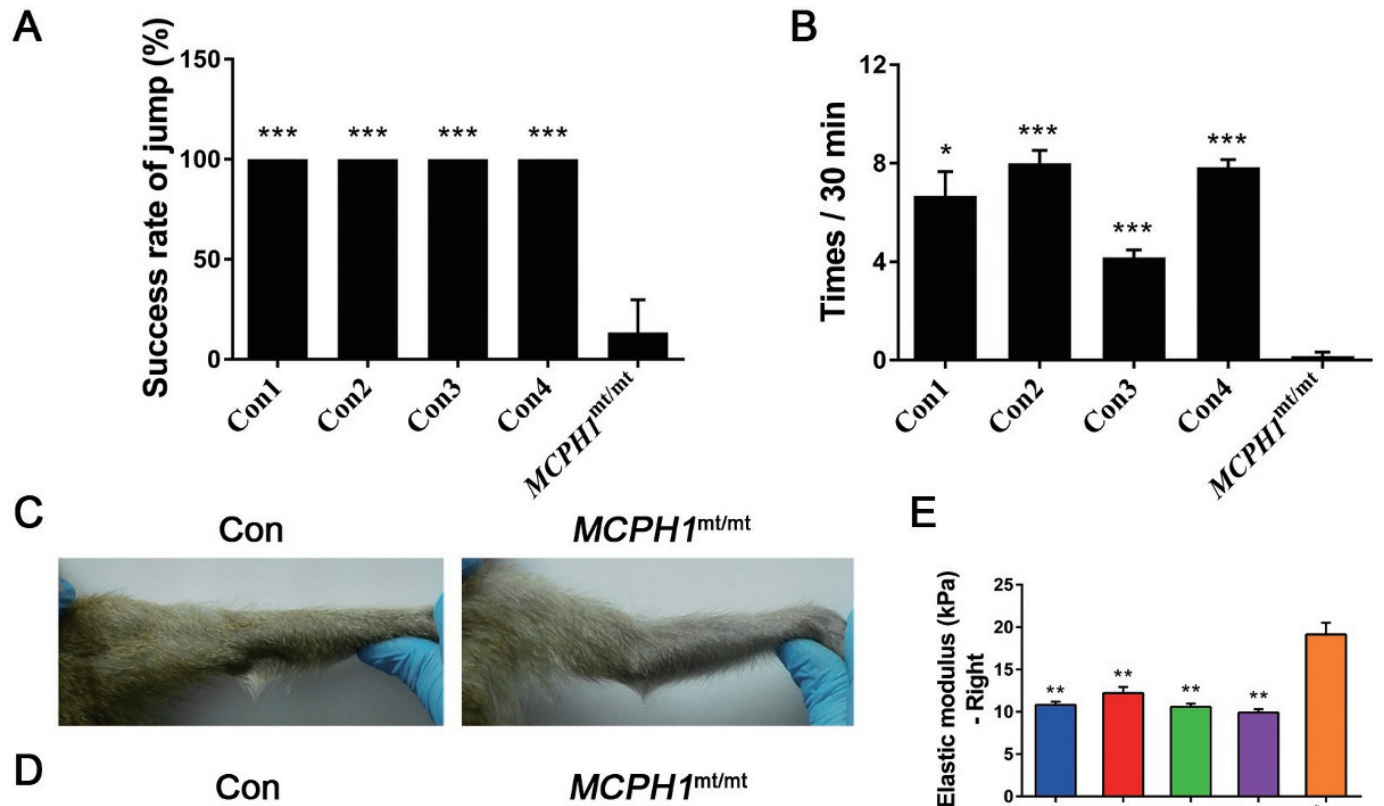

$E$
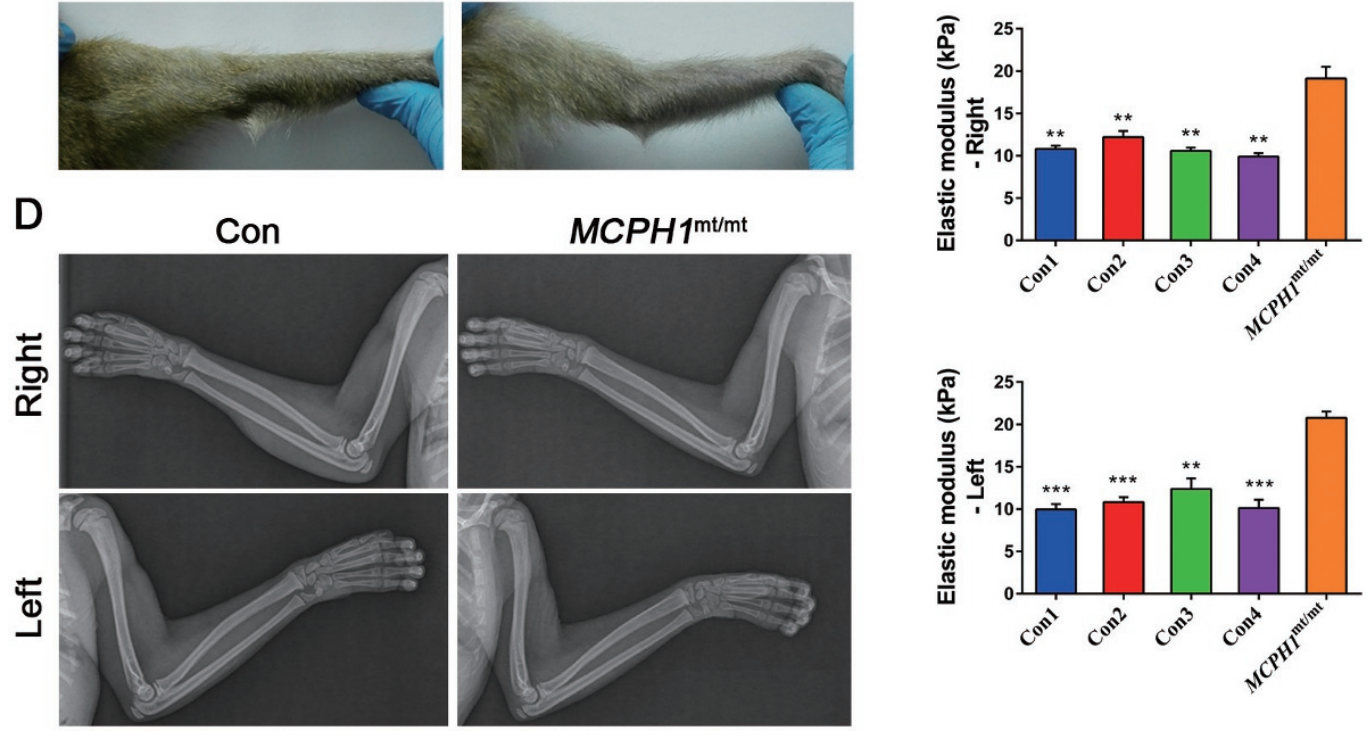

Figure 3 Behavioral and neurological changes in MCPH1 gene mutant monkey. (A) Histogram depicting the jumping success rate of wild-type monkeys and the $M C P H 1^{\mathrm{mt} / \mathrm{mt}}$ monkey in $30 \mathrm{~min}$. The $M C P H 1$ mutant monkey almost always failed to successfully jump up to a higher perch. $n=6$. (B) Histogram depicting the instances of hanging upside down observed for wildtype monkeys and the $M C P H 1^{\mathrm{m} t / \mathrm{mt}}$ monkey in $30 \mathrm{~min} . n=6$. (C) Images of the arms of wild-type monkeys and the MCPH1 mutant monkey. The latter arms are bent and could not be fully extended by external force. (D) X-ray films depicting both arms of wild-type monkeys and the MCPH1 mutant monkey. Radiological examination of the $M C P H 1^{\mathrm{mt} / \mathrm{mt}}$ monkey did not find any bone deformity. (E) Histogram depicting the shear modulus of wild-type monkeys and the $M C P H 1^{\mathrm{mt} / \mathrm{mt}}$ monkey. The biceps brachii of the MCPH1 mutant monkey exhibited significantly greater stiffness than that of the controls. $n=4$. All data are represented as the mean $\pm \mathrm{SEM},{ }^{\star} P<0.05,{ }^{* \star} P<0.01,{ }^{* \star *} P<0.001$ versus the $M C P H 1^{\mathrm{m} / \mathrm{mt}}$ monkey (one-way ANOVA). $M C P H 1^{\mathrm{mt} / \mathrm{mt}}, M C P H 1$ mutant monkey; Con, wild-type monkey.

could reverse the mutant phenotypes of $M C P H 1^{\mathrm{mt} / \mathrm{mt}}$ monkey DFs, suggesting that the mutations in $\mathrm{MCPHI}$ but no other potential OTS are primarily responsible for the phenotype observed in the $M C P H I^{\mathrm{m} t \mathrm{mt}}$ monkey.

\section{Discussion}

Here we used TALEN technology to successfully generate the first $\mathrm{MCPH} 1$ mutant cynomolgus monkey. In monkey embryos, our TALEN pairs exhibited high targeting efficiency with low toxicity as previously reported
$[30,31]$. Using these TALEN pairs, we obtained one live female monkey harboring biallelic $M C P H 1$ mutations without detectable off-target effect. Our study suggest that TALEN technology can be successfully adopted to develop rapid, efficient and precise mutagenesis in cynomolgus monkeys.

The human $M C P H 1$ gene is a recessive gene located on chromosome 8p23. The MCPH1 gene of the cynomolgus monkey is similarly mapped to chromosome 8 . Our target sites are located in exon 2 or 3 , and nearly all of the missense mutations shown to cause primary mi- 
A

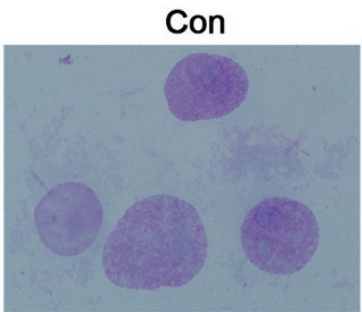

B

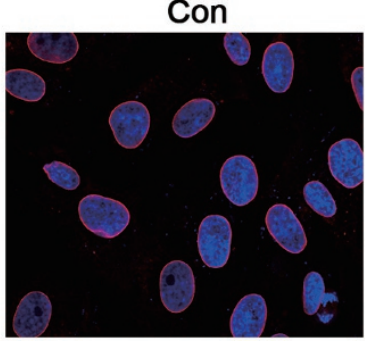

C

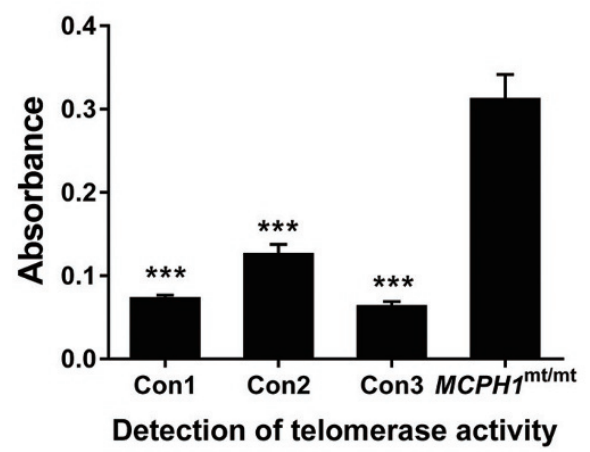

$M C P H 1^{\mathrm{mt} / \mathrm{mt}}$

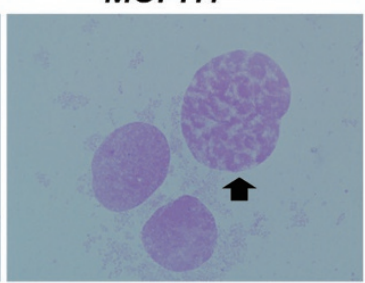

$M C P H 1^{\mathrm{mt} / \mathrm{mt}}$

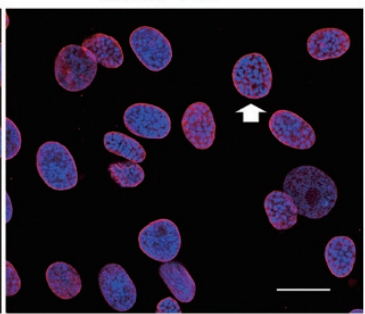

D
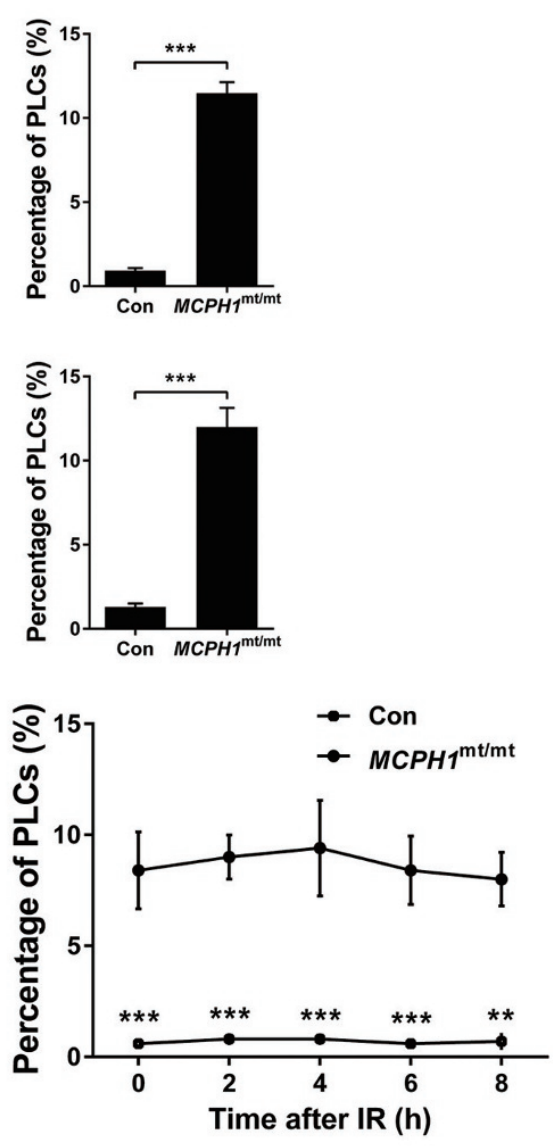

E
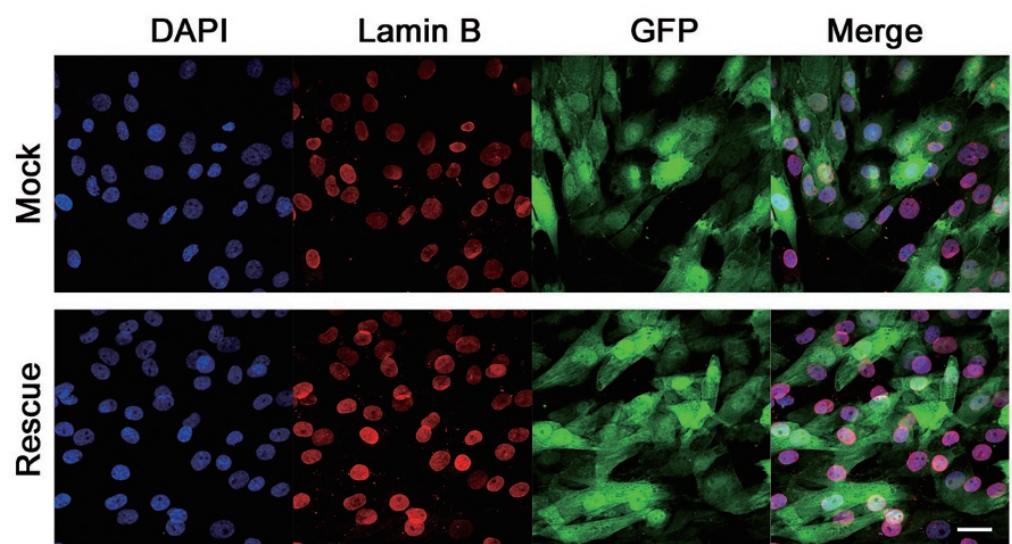

$\mathbf{F}$

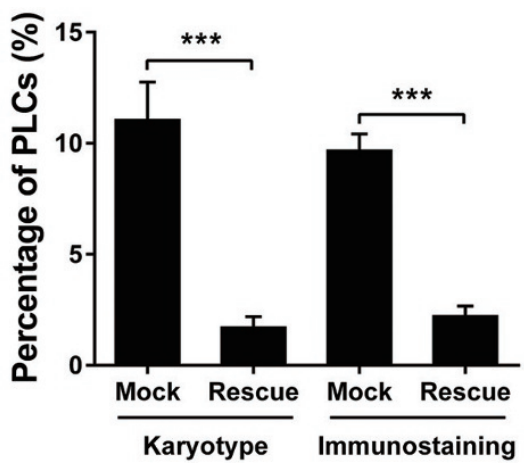

G

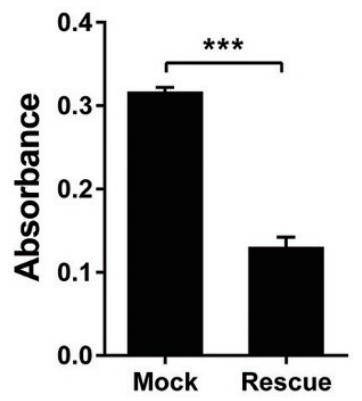

Detection of telomerase activity 
crocephaly are located in exon 2 or $3[9,17,19,20,40$, 45]. These exons encode the evolutionarily conserved $\mathrm{N}$-terminal BRCT1 domain $[39,46]$. Genotype analysis revealed that the TALEN-generated $M C P H 1$ mutant cynomolgus monkey has two types of mutations. One is predicted to generate a truncated protein $(75 \mathrm{aa})$ with impaired function, whereas the other a protein missing one amino acid (p.57QST $>$ HL). The p.57QST $>$ HL mutation is likely to have a functional consequence, as QST is highly conserved among different species (Supplementary information, Figure S4A). Structure modeling of the $\mathrm{N}$-terminal domain of microcephalin suggests the QST to HL mutation may reduce the original three turns of $\alpha$-helix to two turns of $\alpha$-helix (Supplementary information, Figure S4B), thus affecting the protein stability or interaction with other proteins. Richards et al. reported that the $\alpha 2$ region containing QST residues (from p.57 to p.66; Supplementary information, Figure S4A) is one of the important sites for $\mathrm{MCPH1}$ function in preventing abnormal metaphase chromosome condensation [39]. Taken together, p.57QST $>$ HL mutation may significantly disrupt microcephalin structure and function.

Small head circumference is an important clinical index used to evaluate patients with $M C P H 1$ gene defects [47]. The head circumference of patients is in the range of -3 to -11 SD below the means. The head circumference of the $M C P H 1^{\mathrm{mt} / \mathrm{mt}}$ monkey was remarkably smaller (approximately $-5 \mathrm{SD}$ ) than that of the wild-type controls until 12 months of age. Previous studies have reported that $M C P H 1$ mutant patients always exhibit short stature, but the brain size is always significantly more reduced than height $[9,18]$. The $M C P H 1^{\mathrm{mt} / \mathrm{mt}}$ monkey also exhibited a slower growth trajectory, and we then calculated the ratio of OFC to body length of the MCPH1 mutant monkey and control animals. The ratio was $68.2 \%$ in the $M C P H 1^{\mathrm{mt} / \mathrm{mt}}$ monkey, but $73.1 \% \pm 3.0 \%$ in the controls at 12 months of age (mean $\pm \mathrm{SD}, P<0.05$, Student's $t$-test), suggesting the brain size is also significantly more reduced than the length in $M C P H 1$ mutant monkey. MRI revealed that the $M C P H 1^{\mathrm{mt} / \mathrm{mt}}$ monkey had a thin and hypoplastic corpus callosum but no other apparent cerebral malformation. Similarly, MRI scans of some $M C P H 1$ mutant patients have identified malformations of the brain, such as hypoplasia of the frontal lobe and corpus callosum, reduction of the cerebral cortex, and a primitive, coarsened gyral pattern $[18,21]$. In contrast, in most MCPH1 mutant mice, MRI analyses did not reveal any obvious malformation in the brain [26, 27]. Only Gruber and Zhou et al. reported that the cerebral cortex was reduced in their MCPH1 mutant mice [24, 25, 48]. These results indicate that $M C P H 1$ is likely to have a more important role in the brain development of primates than that of mice.

As some $M C P H 1$ mutant patients were reported to display spasticity and delayed motor skills, we hypothesized that the dysmyotonia and body cramping observed in the $M C P H I^{\mathrm{m} t \mathrm{mt}}$ monkey were related to the gene mutation. Spasticity has been connected with a thin corpus callosum in various other diseases $[49,50]$. In a previous report, a $\mathrm{MCPH1}$ mutant patient exhibited agenesis of the genu of the corpus callosum with other cerebral malformations, and she also showed slow developmental progress combined with profound mental retardation [18]. Because brain development continues after birth in primates and the $M C P H 1^{\mathrm{m} t \mathrm{mt}}$ monkey is in the early stage of life, the etiology of these symptoms remains unknown. To our knowledge, no neurological symptom has been reported in a MCPH1 mutant mouse. These prior and present observations provide additional support for our belief that $M C P H 1$ has a more critical role during brain development in primates than in mice.

At the cellular level, PCC is a key discriminating fea-

Figure 4 Cellular phenotype of TALEN-targeted $M C P H 1$ gene mutant monkey. (A) Chromosome preparations of normal and $M C P H 1^{\mathrm{mt} / \mathrm{mt}}$ DFs without prior colchicine treatment. A high proportion of prophase-like cells (black arrow) are present in the $M C P H 1^{\mathrm{mt} / \mathrm{mt}}$ DFs. The histogram depicts the proportion of cells with a prophase-like appearance. (B) Immunofluorescence analysis of lamin $\mathrm{B}$ (red) and DAPI (blue) in normal and $M C P H 1^{\mathrm{mt} / \mathrm{mt}} \mathrm{DFs}$. Many of the $M C P H 1^{\mathrm{mt} / \mathrm{mt}}$ DFs exhibit intact nuclear membranes with prematurely condensed chromosomes. A cell (white arrow) shows a remarkable prophase-like appearance. The histogram shows the proportion of cells with a prophase-like appearance. Bar $=20 \mu \mathrm{m}$. (C) Telomerase enzymatic activity in DFs was assessed using the telomeric repeat amplification protocol (TRAP) versus MCPH1 ${ }^{\text {t/mt }}$ monkey (one-way ANOVA). (D) The proportion of cells with a prophase-like appearance in $M C P H 1^{\mathrm{m} t \mathrm{mt}}$ DFs after ionizing irradiation. (E) Immunofluorescence analysis of lamin B (red) and DAPI (blue) in $M C P H 1^{\mathrm{mt} / \mathrm{mt}}$ DFs. GFP fluorescence reflects the viral infection efficiency. Bar $=20 \mu \mathrm{m}$. (F) The histogram depicts the proportion of cells with a prophase-like appearance among $M C P H 1^{\mathrm{mt} / \mathrm{mt}} \mathrm{DFs}$ transfected with vectors encoding eGFP (Mock) or MCPH1-eGFP (Rescue). "Karyotype" indicates the chromosome preparation experiment, whereas "immunostaining" indicates the immunofluorescence analysis of lamin B. (G) Telomerase enzymatic activity was quantified by TRAP in MCPH1 ${ }^{\mathrm{mt} / \mathrm{mt}}$ DFs transfected with vectors encoding eGFP (Mock) or MCPH1-eGFP (Rescue). All data are represented as the mean \pm SEM, $n=3,{ }^{* *} P<0.01$, ${ }^{* *} P<0.001$ (Student's $t$-test expect C). Con, wild-type monkey; $M C P H 1^{\mathrm{mt} / \mathrm{mt}}, M C P H 1$ mutant monkey; Mock, eGFP expression vector; Rescue, MCPH1-eGFP expression vector; PLCs, prophase-like cells. 
ture presented in human $M C P H 1$ patients [18, 39], and the percentage of PLCs reportedly ranges from $3.3 \%$ to $18 \%$. Here we show that $\sim 11.5 \%$ of the $M C P H I^{\mathrm{m} t \mathrm{mt}} \mathrm{DFs}$ were PLCs, which is significantly higher than in the control. Consistent with the report of Gavvovidis et al. [44], we observed that the DFs of the $M C P H I^{\mathrm{mt} / \mathrm{mt}}$ monkey did not decondense their chromatin following DNA damage (which normally occurs to allow repair). Moreover, cytological studies also verified that $\mathrm{MCPHI}$ acts as a BRCT-repeat-containing inhibitor of TERT expression. The PCC phenotype is reportedly caused by the lack of a functional N-terminal BRCT domain of microcephalin $[39,40]$. Some patients have no significant increase in the fraction of prophase stage chromosomes; their mutations have been mapped in exons 4 and 5 [51, 52]. In our study, the western blot analysis revealed an intermediate level of microcephalin protein expression. Because the antigen used to develop this antibody is located between amino acid 400 and 500 of the canonical sequence according to Uniprot data for microcephalin, a truncated protein (75 aa) cannot be detected, but a protein missing one amino acid (p.57QST $>$ HL) can be detected by the antibody. As we did not observe any wild-type $\mathrm{MCPH1}$ allele and we could observe PCC in this monkey, we presume that the observed protein may be translated from the deletion/missense mutant allele. To further confirm that the observed cellular phenomena were due to the mutation of $M C P H 1$, we ectopically expressed $M C P H 1$ in $M C P H 1^{\mathrm{mt} / \mathrm{mt}}$ monkey DFs and found that this strategy could reverse the mutant phenotypes. Taken together, the in vitro findings demonstrate that the $M C P H 1$ mutant monkey cells possess most of the features of $\mathrm{MCPHI}$ mutant patient cells.

In summary, we have successfully established a MCPH1 mutant cynomolgus monkey model using TALENs. The phenotype of the $M C P H 1^{\mathrm{mt} / \mathrm{mt}}$ monkey closely resembles that of $M C P H 1$ mutant microcephaly in humans, indicating this animal model may provide a new strategy for studying the pathogenesis of microcephaly and the role microcephalin in the evolution of the brain size in primates.

\section{Materials and Methods}

\footnotetext{
Animals

Six healthy female cynomolgus monkeys (age 5-8 years) with regular menstrual cycles were selected as oocyte donors for superovulation, and nine multiparous monkeys were chosen as embryo recipients. Three healthy male cynomolgus monkeys (age 8 to 10 years) with body weights $\sim 8 \mathrm{~kg}$ were selected as sperm donors. All animals were housed at the Blooming Spring Biological Technology Development Co., LTD, which is fully accredited by the Association for Assessment and Accreditation of Laboratory An-
}

imal Care International (AAALAC). The use and care of animals were approved by the Ethics Committee of Sun Yat-sen University.

Sperm and oocyte collection, TALEN injection, and embryo transfer

Monkey semen was collected by rectal probe electro-ejaculation and washed with TL-HEPES. Embryo collection was performed as previously described [53]. Six healthy female cynomolgus monkeys were intramuscularly injected with rhFSH (recombinant human follitropin alfa; GONAL-F, Merck Serono) daily for 8 days, followed by rhCG (recombinant human chorionic gonadotropin alfa; OVIDREL, Merck Serono) on day 9. Oocytes were collected by laparoscopy 33-36 h after rhCG administration $[53,54]$. Metaphase II (MII, first polar body is present) oocytes were cultured in CMRL-1066 medium containing $0.1 \%$ Na-lactate (Sigma, L1375) and subjected to intracytoplasmic sperm injection (ICSI). At $8 \sim 10 \mathrm{~h}$ after ICSI, the zygotes were injected with TALEN mRNAs at 20 or $50 \mathrm{ng} / \mu \mathrm{l}$. The zygotes were transferred into surrogate females at the pronuclear stage or cultured in embryo culture medium-9 (HECM-9) containing 10\% fetal bovine serum (FBS; Hyclone Laboratories, SH30088.02) at $37{ }^{\circ} \mathrm{C}$ in $5 \%$ $\mathrm{CO}_{2}$. Early pregnancy diagnosis was performed by ultrasonography $\sim 30$ days after embryo transfer. Both clinical pregnancy and the number of fetuses were confirmed by fetal cardiac activity and the presence of a yolk sac, as detected by ultrasonography [54].

\section{Cell culture}

DFs derived from the $M C P H I^{\mathrm{mt} / \mathrm{mt}}$ monkey and wild-type animals were established from monkey ears and propagated in standard condition. Cells were grown in Dulbecco's modified Eagle's medium (DMEM)/high glucose (Hyclone) containing 10\% FBS and maintained at $37{ }^{\circ} \mathrm{C}$ in air containing $5 \% \mathrm{CO}_{2}$.

COS-7 cells were cultured in DMEM/high glucose supplemented with $10 \%$ FBS and $1 \%(\mathrm{w} / \mathrm{v})$ penicillin/streptomycin (Invitrogen/Gibco). TALEN expression plasmids were transfected into COS-7 cells by electroporation (BioRad Gene Pulser XL), and an eGFP-encoding plasmid was used as a control. Cells were collected for further analysis at $72 \mathrm{~h}$ post electroporation.

\section{TALEN target site design and unit assembly}

TALEN target sites were designed and constructed according to the instruction provided with the e-TALEN kit (Viewsolid Biotech). Briefly, units were assembled to construct the TALE repeats according to the target sequences, using several rounds of digestion/ligation. The constructed repetitive TALE fragments were then digested and inserted into the pCS2-eTALEN-T vector.

\section{Production of TALEN $m R N A s$}

TALEN plasmids $(5 \mu \mathrm{g}$; pCS2-eTALEN-T) were linearized by NotI digestion in NEBuffer 2 (NEB) for $6 \mathrm{~h}$ at $37^{\circ} \mathrm{C}$, purified with a PCR purification kit (Qiagen), eluted with RNase-free water and diluted to a final concentration of $\sim 200 \mathrm{ng} / \mu \mathrm{l}$. In vitro transcription was performed using an SP6 mMESSAGE mMACHINE Kit (Ambion) according to the manufacturer's instruction. The $10-\mu 1$ reaction mixture, which contained $5 \mu 1$ of $2 \times$ NTP/CAP, $1 \mu l$ of $10 \times$ reaction buffer, $1 \mu 1$ of SP6 enzyme mix and $500 \mathrm{ng}$ of linear template DNA, was incubated at $37^{\circ} \mathrm{C}$ for $3 \mathrm{~h}$. The TALENs were recovered by lithium chloride precipitation. Then, the left and right TALEN RNAs were mixed at a ratio of 1:1 ( $50 \mathrm{ng} / \mu \mathrm{l}$ each), and 
the mixture was stored at $-80{ }^{\circ} \mathrm{C}$.

\section{T7EN1 cleavage assay and genomic DNA sequencing}

Genomic DNA was isolated from cultured embryos using a GenomePlex Single Cell Whole Genome Amplification Kit (Sigma) according to the manufacturer's protocol. Genomic DNA was isolated from tissues using a DNA extraction kit (Qiagen) according to the manufacturer's instructions. PCR amplicons, including nuclease target sites or OTSs, were generated using ExTaq polymerase (Takara) and the primers listed in Supplementary information, Table S2. The obtained PCR products ranged in the size from 300 to $600 \mathrm{bp}$ and were purified with a PCR purification kit. Briefly, the T7EN1 assay was performed as follows. Using a thermocycler, we denatured and annealed the PCR products in $1 \times$ NEBuffer 2 (NEB) to form heteroduplex DNA, which was then digested with T7EN1 (NEB) for $15 \sim 25 \mathrm{~min}$ at $37{ }^{\circ} \mathrm{C}$ and analyzed by $2 \%$ agarose gel electrophoresis. The cleavage bands indicated modification of the target sites. Purified PCR products were also ligated into the pMD19-T vector (Takara), transformed into competent $E$. coli strain DH5 $\alpha$ (Takara) and subjected to sequencing analysis.

\section{Off-target analysis}

Using TALENoffer, we selected 13 intronic loci and 18 exonic loci as potential OTS (Supplementary information, Table S1). The potential OTSs were amplified from monkey genomic DNA using specific primers (Supplementary information, Table S2). Then, $300 \mathrm{ng}$ of each purified PCR product mixed with wild-type PCR product was digested with T7EN1 for 15 25 min, and the treated products were recovered by $2 \%$ agarose gel electrophoresis.

\section{Physical measurements}

Four age-matched wild-type female cynomolgus monkeys were selected as normal controls. All monkeys were raised under the same condition in the same room. Physical measurements, including OFC, body weight, body length, tail length and chest circumference, were obtained monthly. Here we present data representing $\sim 2$-month intervals.

\section{MRI measurements and calculation of brain volume}

Brain images of monkeys were acquired using a GE Discovery MR750 3.0T scanner (GE, Milwaukee, USA) with an HD wrist array coil. Monkeys were initially anesthetized with $3 \sim 5 \mathrm{mg} / \mathrm{kg}$ of Telazol, and then intubated and maintained under anesthesia using $1.0 \% \sim 1.5 \%$ isoflurane mixed with $\mathrm{O}_{2}$. All major physiological parameters were monitored continuously and maintained within normal ranges. With respect to the detailed sequence parameters of the MRI scans, we obtained the following: SAG T2 Propeller FRFSE-weighted images (FRFSE, TE $=98 \mathrm{~ms}$, TR $=9578 \mathrm{~ms}$, FOV $=120 \mathrm{~mm}$, matrix $=384 \times 384$, thickness $=2 \mathrm{~mm}$, spacing $=0.2 \mathrm{~mm}, \mathrm{FA}=142, \mathrm{BW}=83.3 \mathrm{KHz}, \mathrm{ETL}=32, \mathrm{NEX}=1.5)$; Ax T2 Propeller FSE-weighted images $(\mathrm{FSE}, \mathrm{TE}=89 \mathrm{~ms}, \mathrm{TR}=10$ $219 \mathrm{~ms}, \mathrm{FOV}=120 \mathrm{~mm}$, matrix $=320 \times 320$, thickness $=2 \mathrm{~mm}$, spacing $=0.2 \mathrm{~mm}, \mathrm{FA}=142, \mathrm{BW}=62.5 \mathrm{KHz}, \mathrm{ETL}=32, \mathrm{NEX}=$ 2); and Ax 3D T1 BRAVO-weighted images (BRAVO, TE $=3.7$ $\mathrm{ms}, \mathrm{TR}=8.7 \mathrm{~ms}, \mathrm{TI}=450, \mathrm{FOV}=128 \mathrm{~mm}$, matrix $=256 \times 256$, thickness $=0.5 \mathrm{~mm}, \mathrm{FA}=12, \mathrm{BW}=31.25 \mathrm{KHz}, \mathrm{NEX}=1)$. Regional and TBVs were calculated using the FSL Brain Extraction Tool (FSL5.0) and DPABI V1.2 141101 software [55, 56].
X-ray

A dual-detector X-ray unit (DiDiEleva01; Philips, Holland) was used to acquire radiographs at $10 \mathrm{~mA}$ with $60 \mathrm{kV}$, using a grid and an exposure time of $0.2 \mathrm{~s}$. An SRO 33100 ROT 360 X-ray tube (Philips) was used. The focal spot was $0.7 \times 0.7 \mathrm{~mm}$, and the focal distance for all images was $110 \mathrm{~mm}$. The DigitalDiagnost (2.1.4 V22.13.567 2012) software program was used for radiology.

\section{Behavioral assessment}

For behavioral assessments, the $M C P H 1^{\mathrm{mt} / \mathrm{mt}}$ monkey was caged with each of four wild-type monkeys in turn and recorded by a camera at a regular daily time when the monkeys were 8-month old. Videos were collected and divided into a period of $30 \mathrm{~min}$ and six random videos were selected for each monkey. They were next analyzed by two raters in a blinded manner. The following types of behavioral movements were scored: the times of each monkey jump (jump from the bottom to the top using only its legs) and success, as well as the times of each monkey hang upside down in $30 \mathrm{~min}$.

\section{Ultrasound elastography}

The forearm of each anesthetized monkey was placed in a $90^{\circ}$ flexed position, and ultrasound and shear-wave ultrasound elastographic measurements of the biceps brachii were performed using an Aixplorer ultrasound scanner (Supersonic Imagine; Aixen-Provence, France) coupled with a linear transducer array (415 MHz, SuperLinear 15-4; Vermon, Tours, France). The biceps brachii tendon elasticity values were measured within a defined region of interest at the thickest point. All measurements were performed by two ultrasound specialist radiologists and reported in kilopascals.

\section{Chromosome preparation and PLC assay}

Metaphase spreads of DFs were performed using standard diagnostic laboratory procedures. Slides were stained with fresh $10 \%$ Giemsa stain for $10 \mathrm{~min}$. Approximately 1000 nuclei per experiment were counted in three independent experiments for each group, and the percentage of prophase-like nuclei was determined with respect to total nuclei.

\section{Western blot analysis}

Total proteins were extracted from DFs and PBMCs, and analyzed as previously described. Equal amounts of soluble protein were separated via sodium dodecyl sulphate-polyacrylamide gel electrophoresis using 10\% Tris-glycine mini-gels and transferred onto a nitrocellulose membrane (Bio-Rad). Rabbit anti-BRIT1 (1:1 000, \#4120; Cell Signaling) and rabbit anti-GAPDH (1:5 000, \#2118; Cell Signaling) were used as the primary antibodies. Chemiluminescence (Amersham) was used to visualise the protein bands on X-ray films (Kodak). All of the western blot exposures were within the linear detection range.

\section{Telomeric repeat amplification assay}

Cellular telomerase activity was measured by telomerase repeat sequence amplification-enzyme linked immunosorbent assay using the TeloTAGGGTelomerase PCR ELISA kit (11854666910; Roche) according to the manufacturer's instructions. Sample absorbance was measured with a Model 550 Microplate Reader (Bio$\mathrm{Rad}, \mathrm{USA}$ ) at 450/690 $\mathrm{nm}$ within $30 \mathrm{~min}$ after addition of the stop 
reagent.

\section{IR and immunofluorescence staining}

Logarithmically proliferating DFs were irradiated using an X-ray source $(\mathrm{Ua}=160 \mathrm{kV} ; \mathrm{I}=25 \mathrm{~mA}$; dose rate, $1.02 \mathrm{~Gy} / \mathrm{min}$; Rs2000; Rad Source). For immunofluorescence, DFs grown on coverslips were fixed with $4 \%(\mathrm{v} / \mathrm{v})$ paraformaldehyde in phosphate-buffered saline ( $\mathrm{pH}$ 7.4) for $15 \mathrm{~min}$ at room temperature, permeabilized in $0.1 \%(\mathrm{v} / \mathrm{v})$ Triton X-100 and blocked using 2\% $(\mathrm{w} / \mathrm{v})$ bovine serum albumin. The cells were then incubated at 4 ${ }^{\circ} \mathrm{C}$ overnight with anti-lamin B (sc-6216; Santa Cruz) in 2\% (w/v) bovine serum albumin. The cells were washed and incubated with Alexa Fluor 488/Alexa Fluor 594-conjugated goat anti-mouse/ anti-rabbit secondary antibody (Invitrogen) at room temperature for $1 \mathrm{~h}$ in the dark. Nuclei were counterstained with DAPI (Sigma). The results were analyzed by direct observation under laser confocal microscopy (LSM 780; Carl Zeiss) and using ZEN 2009 Light Edition software (Carl Zeiss).

\section{Statistical analysis}

Statistical analyses were performed with SPSS software (version 17.0; SPSS Institute). Significant differences between means were assessed by Student's $t$-test or one-way ANOVA. Statistical significance was assumed at $P<0.05$, and all $P$-values are presented as two-tailed results.

\section{Acknowledgments}

This work was supported by the National Basic Research Program of China (2012CBA01302), the National Natural Science Foundation of China (81425016, 31301219 and 81570593), the Fundamental Research Funds for the Central Universities (15ykjc01c), PhD Programs Foundation of Ministry of Education of China (20130171120061), the Key Scientific and Technological Projects of Guangdong Province (2014B020226002, 2014B020228003, 2014B020225007, 2015B020228002 and 2016B030229002), Key Scientific and Technological Program of Guangzhou City (201400000003-3, 201508020262, 201300000089 and 2010U1-E00551), Guangdong Province Universities and Colleges Pearl River Scholar Funded Scheme (GDUPS, 2013) and Pearl River S\&T Nova Program of Guangzhou (2014J2200037).

\section{Author Contributions}

QK and WL designed experiments, performed research, interpreted data and wrote the manuscript. XL performed research and interpreted data and wrote the manuscript. $\mathrm{CH}, \mathrm{HZ}, \mathrm{YM}, \mathrm{DX}, \mathrm{TW}$ and FFM contributed to embryo manipulation. LH, XF, ZK and HX performed MRI and X-ray analysis. KL and JR performed ultrasound elastography. WH performed bioinformatics analysis. ZC, XS and XL contributed to chromosome preparation. MZ contributed to protein structure prediction and analysis. FZ and JX provided TALENs. BTL. edited the manuscript. QZ, SH and APX designed and supervised research, interpreted data and wrote the manuscript. QK, WL and XL contributed equally to this work.

\section{Competing Financial Interests}

The authors declare no competing financial interests.

\section{References}

1 Nakano T, Ando S, Takata N, et al. Self-formation of optic cups and storable stratified neural retina from human ESCs. Cell Stem Cell 2012; 10:771-785.

2 Lancaster MA, Renner M, Martin CA, et al. Cerebral organoids model human brain development and microcephaly. $\mathrm{Na}$ ture 2013; 501:373-379.

3 Herculano-Houzel S. The human brain in numbers: a linearly scaled-up primate brain. Front Hum Neurosci 2009; 3:31.

4 Zecevic N, Rakic P. Development of layer I neurons in the primate cerebral cortex. J Neurosci 2001; 21:5607-5619.

5 Games D, Adams D, Alessandrini R, et al. Alzheimer-type neuropathology in transgenic mice overexpressing V717F $\beta$-amyloid precursor protein. Nature 1995; 373:523-527.

6 Kishi N, Sato K, Sasaki E, Okano H. Common marmoset as a new model animal for neuroscience research and genome editing technology. Dev Growth Differ 2014; 56:53-62.

7 Chan AW. Transgenic nonhuman primates for neurodegenerative diseases. Reprod Biol Endocrinol 2004; 2:39.

8 Belmonte JC, Callaway EM, Churchland P, et al. Brains, Genes, and Primates. Neuron 2015; 86:617-631.

9 Pulvers JN. MCPH1: a window into brain development and evolution. Front Cell Neurosci 2015; 9:92.

10 Woods CG, Parker A. Investigating microcephaly. Arch Dis Child 2013; 98:707-713.

11 Woods CG, Bond J, Enard W. Autosomal recessive primary microcephaly $(\mathrm{MCPH})$ : a review of clinical, molecular, and evolutionary findings. Am J Hum Genet 2005; 76:717-728.

12 Jackson AP, McHale DP, Campbell DA, et al. Primary autosomal recessive microcephaly (MCPH1) maps to chromosome 8p22-pter. Am J Hum Genet 1998; 63:541-546.

13 Faheem M, Naseer MI, Rasool M, et al. Molecular genetics of human primary microcephaly: an overview. BMC Med Genomics 2015; 8 (Suppl 1):S4.

14 Evans PD, Anderson JR, Vallender EJ, Choi SS, Lahn BT. Reconstructing the evolutionary history of microcephalin, a gene controlling human brain size. Hum Mol Genet 2004; 13:11391145.

15 Wang YQ, Su B. Molecular evolution of microcephalin, a gene determining human brain size. Hum Mol Genet 2004; 13:1131-1137.

16 Evans PD, Gilbert SL, Mekel-Bobrov N, et al. Microcephalin, a gene regulating brain size, continues to evolve adaptively in humans. Science 2005; 309:1717-1720.

17 Jackson AP, Eastwood H, Bell SM, et al. Identification of microcephalin, a protein implicated in determining the size of the human brain. Am J Hum Genet 2002; 71:136-142.

18 Neitzel H, Neumann LM, Schindler D, et al. Premature chromosome condensation in humans associated with microcephaly and mental retardation: a novel autosomal recessive condition. Am J Hum Genet 2002; 70:1015-1022.

19 Trimborn M, Richter R, Sternberg N, et al. The first missense alteration in the $\mathrm{MCPH} 1$ gene causes autosomal recessive microcephaly with an extremely mild cellular and clinical phenotype. Hum Mutat 2005; 26:496.

20 Darvish H, Esmaeeli-Nieh S, Monajemi GB, et al. A clinical and molecular genetic study of 112 Iranian families with primary microcephaly. J Med Genet 2010; 47:823-828. 
21 Pfau RB, Thrush DL, Hamelberg E, et al. MCPH1 deletion in a newborn with severe microcephaly and premature chromosome condensation. Eur J Med Genet 2013; 56:609-613.

22 Ghani-Kakhki M, Robinson PN, Morlot S, et al. Two missense mutations in the primary autosomal recessive microcephaly gene $\mathrm{MCPHI}$ disrupt the function of the highly conserved N-terminal BRCT domain of microcephalin. Mol Syndromol 2012; 3:6-13.

23 Trimborn M, Bell SM, Felix C, et al. Mutations in microcephalin cause aberrant regulation of chromosome condensation. Am J Hum Genet 2004; 75:261-266.

24 Chen J, Ingham N, Clare S, et al. Mcph1-deficient mice reveal a role for MCPH1 in otitis media. PLoS One 2013; 8:e58156.

25 Gruber R, Zhou Z, Sukchev M, et al. MCPH1 regulates the neuroprogenitor division mode by coupling the centrosomal cycle with mitotic entry through the Chk1-Cde25 pathway. Nat Cell Biol 2011; 13:1325-1334.

26 Trimborn M, Ghani M, Walther DJ, et al. Establishment of a mouse model with misregulated chromosome condensation due to defective Mcph1 function. PLoS One 2010; 5:e9242.

27 Liang Y, Gao H, Lin S, et al. BRIT1/MCPH1 is essential for mitotic and meiotic recombination DNA repair and maintaining genomic stability in mice. PLoS Genet 2010; 6:e1000826.

28 Yang S, Cheng P, Banta $\mathrm{H}$, et al. Towards a transgenic model of Huntington's disease in a non-human primate. Nature 2008; 453:921-924.

29 Liu Z, Li X, Zhang JT, et al. Autism-like behaviours and germline transmission in transgenic monkeys overexpressing MeCP2. Nature 2016; 530:98-102.

30 Liu H, Chen Y, Niu Y, et al. TALEN-mediated gene mutagenesis in rhesus and cynomolgus monkeys. Cell Stem Cell 2014; 14:323-328.

31 Liu Z, Zhou X, Zhu Y, et al. Generation of a monkey with $M E C P 2$ mutations by TALEN-based gene targeting. Neurosci Bull 2014; 30:381-386.

32 Niu Y, Shen B, Cui Y, et al. Generation of gene-modified cynomolgus monkey via Cas9/RNA-mediated gene targeting in one-cell embryos. Cell 2014; 156:836-843.

33 Wan H, Feng C, Teng F, et al. One-step generation of p53 gene biallelic mutant cynomolgus monkey via the CRISPR/ Cas system. Cell Res 2014; 25:258-261.

34 Mussolino C, Morbitzer R, Lutge F, et al. A novel TALE nuclease scaffold enables high genome editing activity in combination with low toxicity. Nucleic Acids Res 2011; 39:92839293.

35 Hockemeyer D, Wang H, Kiani S, et al. Genetic engineering of human pluripotent cells using TALE nucleases. Nat Biotechnol 2011; 29:731-734.

36 Grau J, Boch J, Posch S. TALENoffer: genome-wide TALEN off-target prediction. Bioinformatics 2013; 29:2931-2932.

37 Ghani-Kakhki M, Robinson PN, Morlot S, et al. Two missense mutations in the primary autosomal recessive microcephaly gene $\mathrm{MCPH} 1$ disrupt the function of the highly conserved N-terminal BRCT domain of microcephalin. Mol Syndromol 2012; 3:6-13.

38 Lacourpaille L, Hug F, Nordez A. Influence of passive muscle tension on electromechanical delay in humans. PLoS One 2013; 8:e53159.

39 Richards MW, Leung JWC, Roe SM, et al. A Pocket on the surface of the N-terminal BRCT domain of Mcph1 is required to prevent abnormal chromosome condensation. $J$ Mol Biol 2010; 395:908-915.

40 Leung JW, Leitch A, Wood JL, et al. SET nuclear oncogene associates with microcephalin/MCPH1 and regulates chromosome condensation. J Biol Chem 2011; 286:21393-21400.

41 Lin S, Elledge SJ. Multiple tumor suppressor pathways negatively regulate telomerase. Cell 2003; 113:881-889.

42 Shi L, Li M, Su B. MCPH1/BRIT1 represses transcription of the human telomerase reverse transcriptase gene. Gene 2012; 495:1-9.

43 Rieder CL, Cole RW. Entry into mitosis in vertebrate somatic cells is guarded by a chromosome damage checkpoint that reverses the cell cycle when triggered during early but not late prophase. J Cell Biol 1998; 142:1013-1022.

44 Gavvovidis I, Pohlmann C, Marchal JA, et al. MCPH1 patient cells exhibit delayed release from DNA damage-induced G2/ M checkpoint arrest. Cell Cycle 2010; 9:4893-4899.

45 Hosseini MM, Tonekaboni SH, Papari E, et al. A novel mutation in $\mathrm{MCPH1}$ gene in an Iranian family with primary microcephaly. J Pak Med Assoc 2012; 62:1244-1247.

46 Shi L, Li M, Lin Q, Qi X, Su B. Functional divergence of the brain-size regulating gene $M C P H 1$ during primate evolution and the origin of humans. BMC Biol 2013; 11:62.

47 Mahmood S, Ahmad W, Hassan MJ. Autosomal recessive primary microcephaly $(\mathrm{MCPH})$ : clinical manifestations, genetic heterogeneity and mutation continuum. Orphanet $J$ Rare Dis 2011; 6:39.

48 Zhou Z, Tapias A, Bruhn C, et al. DNA damage response in microcephaly development of MCPH1 mouse model. DNA Repair 2013; 12:645-655.

49 Ma J, Xiong L, Chang Y, et al. Novel mutations c. [5121 5122insAG] $+[6859 \mathrm{C}>\mathrm{T}]$ of the $S P G 11$ gene associated with cerebellum hypometabolism in a Chinese case of hereditary spastic paraplegia with thin corpus callosum. Parkinsonism Relat Disord 2014; 20:256-259.

50 Heimer G, Marek-Yagel D, Eyal E, et al. SLC1A4 mutations cause a novel disorder of intellectual disability, progressive microcephaly, spasticity and thin corpus callosum. Clin Genet 2015; 88:327-335.

51 Farooq M, Baig S, Tommerup N, Kjaer KW. Craniosynostosis-microcephaly with chromosomal breakage and other abnormalities is caused by a truncating MCPH1 mutation and is allelic to premature chromosomal condensation syndrome and primary autosomal recessive microcephaly type 1 . Am J Med Genet Part A 2010; 152A:495-497.

52 Ghafouri-Fard S, Fardaei M, Gholami M, Miryounesi M. A case report: Autosomal recessive microcephaly caused by a novel mutation in MCPH1 gene. Gene 2015; 571:149-150.

53 Niu Y, Yu Y, Bernat A, et al. Transgenic rhesus monkeys produced by gene transfer into early-cleavage-stage embryos using a simian immunodeficiency virus-based vector. Proc Natl Acad Sci USA 2010; 107:17663-17667.

54 Chen Y, Niu Y, Yang S, et al. The available time window for embryo transfer in the rhesus monkey (Macaca mulatta). Am J Primatol 2012; 74:165-173.

55 Smith SM, Jenkinson M, Woolrich MW, et al. Advances in functional and structural MR image analysis and implementation as FSL. Neuroimage 2004; 23 (Suppl 1):S208-S219. 
56 Jenkinson M, Beckmann CF, Behrens TE, Woolrich MW, Smith SM. FSL. Neuroimage 2012; 62:782-790.

(Supplementary information is linked to the online version of the paper on the Cell Research website.) cc)(1) $\Theta$ This work is licensed under a Creative Commons Attribution-NonCommercial-NoDerivs 4.0 Unported License. The images or other third party material in this article are included in the article's Creative Commons license, unless indicated otherwise in the credit line; if thematerial is not included under the Creative Commons license, users will need to obtain permission from the license holder to reproduce the material. To view a copy of this license, visit http://creativecommons.org/licenses/by-nc-nd/4.0/

(C) The Author(s) 2016 